\title{
A Blind Recognition Algorithm of Satellite Communication Modulation Mode Combining Characteristic Parameters and Quadruplicate Spectrum
}

\author{
Liu Yan ${ }^{1, a}$, Cui Qian ${ }^{2, b}$, Xia Jianrong ${ }^{1, c}$ and Qiao Yi $^{1}$ \\ ${ }^{1}$ Beijing Aerospace Control Center, Beijing 102206, China; \\ ${ }^{2}$ Xi'An Space Star Technology Enterprise (Group) Co. 710061, China; \\ avivianliuyan@gmail.com, ${ }^{b}$ cuiqian198611@163.com, ${ }^{c}$ liuhua_xapu@163.com
}

Keywords: modulation mode recognition, high-order cumulants, characteristic parameters, high-order spectrum.

\begin{abstract}
Modulation mode recognition has always been a major subject for domestic and overseas research scholars engaged in the receiving processing of satellite signals. The paper analyzed the high-order cumulants, characteristic parameters, square spectrum and quadruplicate spectrum of 5 most common modulation modes used in satellite communication. Meanwhile, in consideration of the situation that the algorithm in engineering realization should not be too complex, a recognition algorithm of modulation mode which combines characteristic parameter and high-order spectrum. The simulation results showed that when signal to noise ratio was bigger than $10 \mathrm{~dB}$, the recognition rate of this method reached $97 \%$ without any requirement for priori knowledge.
\end{abstract}

\section{Introduction}

Satellite signals is usually divided into three categories: telemetry, remote control and data transmission. Commonly used satellite signals include BPSK, QPSK, OQPSK, MSK, 8PSK, 16QAM and APSK signals. Under the circumstance of non-cooperative communications, effective monitoring and recognition for communication signals have significant application value in both civilian and military fields.

Modulation mode recognition is mainly classified into two kinds: decision theory method based on likelihood ratio and statistic pattern recognition method based on characteristic parameters[1]. In terms of the decision theory method based on likelihood ratio, due to the existence of unknown parameters, the calculation expression of likelihood ratio function is very complex, and the large calculated amount is difficult to deal with. In terms of the statistic pattern recognition method based on characteristic parameters, it is very difficult to find perfectly ideal characteristic parameters due to the limit of measurement difficulty, small characteristic difference and characteristic parameters' noise sensitivity.

Secondly, the mostly commonly used second-order statistic detection methods for signal processing has certain shortcomings: they cannot recognize non-minimum phase system and are sensitive to additive noise[2]. Besides, when power spectrum analysis is carried out for random signals, it is very difficult to avoid the influence of background noise. In theory, high-order cumulant can completely suppress all Gaussian noise and can provide more information than second-order statistics.

Due to above-mentioned problems, this paper combined high-order cumulant, characteristic parameters and high-order spectrum[3] to carry out recognition for modulation mode and software simulation. The simulation results showed that the algorithm can distinguish the five modulation modes of BPSK, 8PSK, QPSK, 16QAM and 16APSK. The algorithm is simple and has relatively high anti-nose performance and relatively high recognition accuracy. 


\section{High-order cumulant}

\section{Definition of High-order Cumulant[4].}

Second-order cumulant:

$\mathrm{C}_{20}=\mathrm{E}[\mathrm{y}(\mathrm{n}) \cdot \mathrm{y}(\mathrm{n})]$

$\mathrm{C}_{20}=\mathrm{E}\left[\mathrm{y}(\mathrm{n}) \cdot \mathrm{y}^{*}(\mathrm{n})\right]$

Fourth-order cumulant:

$\mathrm{C}_{40}=\operatorname{cum}[\mathrm{y}(\mathrm{n}), \mathrm{y}(\mathrm{n}), \mathrm{y}(\mathrm{n}), \mathrm{y}(\mathrm{n})]=\mathrm{E}\left[\mathrm{y}^{4}(\mathrm{n})\right]-3 \mathrm{C}_{20}^{2}$

$\mathrm{C}_{41}=\operatorname{cum}\left[\mathrm{y}(\mathrm{n}), \mathrm{y}(\mathrm{n}), \mathrm{y}(\mathrm{n}), \mathrm{y}^{*}(\mathrm{n})\right]=\mathrm{E}\left[\mathrm{y}(\mathrm{n}) \cdot \mathrm{y}(\mathrm{n}) \cdot \mathrm{y}(\mathrm{n}) \cdot \mathrm{y}^{*}(\mathrm{n})\right]-3 \mathrm{C}_{20} \mathrm{C}_{21}$

$\mathrm{C}_{42}=\operatorname{cum}\left[\mathrm{y}(\mathrm{n}), \mathrm{y}(\mathrm{n}), \mathrm{y}^{*}(\mathrm{n}), \mathrm{y}^{*}(\mathrm{n})\right]=\mathrm{E}\left[\left.\mathrm{y}(\mathrm{n})\right|^{4}\right]-\left|\mathrm{C}_{20}\right|^{2}-2 \mathrm{C}_{21}^{2}$

Sixth-order cumulant:

$\mathrm{C}_{63}=\mathrm{E}\left[|\mathrm{y}(\mathrm{n})|^{6}\right]-9 \mathrm{C}_{42} \cdot \mathrm{C}_{21}-6 \mathrm{C}_{21}^{3}$

The following are theoretical value s of high-order cumulants:

Table 1 Average and normalized theoretical values of modulation signal's cumulants of different orders

\begin{tabular}{ccccccc}
\hline Modulation mode & $\left|\mathrm{C}_{20}\right|$ & $\left|\mathrm{C}_{21}\right|$ & $\left|\mathrm{C}_{40}\right|$ & $\left|\mathrm{C}_{41}\right|$ & $\left|\mathrm{C}_{42}\right|$ & $\left|\mathrm{C}_{63}\right|$ \\
\hline BPSK & $\mathrm{E}$ & $\mathrm{E}$ & $\mathrm{E}^{2}$ & $2 \mathrm{E}^{2}$ & $2 \mathrm{E}^{2}$ & $13 \mathrm{E}^{3}$ \\
8PSK & 0 & $\mathrm{E}$ & 0 & 0 & $\mathrm{E}^{2}$ & $4 \mathrm{E}^{3}$ \\
QPSK & 0 & $\mathrm{E}$ & $\mathrm{E}^{2}$ & 0 & $\mathrm{E}^{2}$ & $4 \mathrm{E}^{3}$ \\
16QAM & 0 & $\mathrm{E}$ & $0.68 \mathrm{E}^{2}$ & 0 & $0.68 \mathrm{E}^{2}$ & $2.08 \mathrm{E}^{3}$ \\
16APSK & 0 & $\mathrm{E}$ & 0 & 0 & $0.78 \mathrm{E}^{2}$ & $2.61 \mathrm{E}^{3}$ \\
\hline
\end{tabular}

Definition of Characteristic Parameters[5].

$$
\begin{aligned}
& \mathrm{F} 1=\frac{\left|\mathrm{C}_{40}\right|}{\left|\mathrm{C}_{42}\right|} \\
& \mathrm{F} 2=\mathrm{C}_{63}^{2} / \mathrm{C}_{42}^{3}
\end{aligned}
$$

By means of matlab simulation, the variation curve of characteristic parameters of modulation signals following symbol rate is as follows:
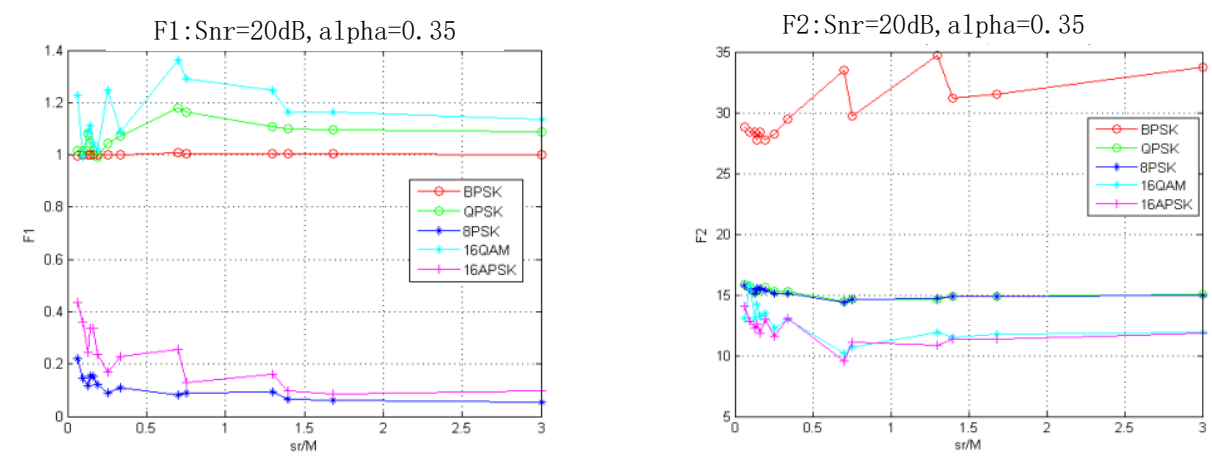

Fig.1 Variation curve of characteristic parameters F1 and F2 following symbol rate

From Figure 1 it is possible to see that signal modulation modes can be divided into 2 categories by using F1. The modulation characteristic parameters of BPSK, QPSK AND 16QAM meet $|\mathrm{F} 1| 2>0.5$, and the modulation of 8PSK AND 16APSK meets $|\mathrm{F} 1| 2<0.5$.

BPSK can be distinguished by means of using F2 value $|\mathrm{F} 2| 2>20$.

\section{The Ratio between EnvelopeVariance and Mean Value}

From the characteristics of time domain, the five modulation modes can be divided into two categories: constant envelope modulation and non-constant modulation. BPSK, QPSK AND 8PSK belong to constant envelope, while 16QAM and 16APSK belong to non-constant modulation. Therefore, the two categories of signals can be distinguished based on envelope characteristics. In theory, the ratio between MPSK's envelope variance and mean value is 0 , while the ratio of 16QAM's and 16APSK's envelope variances and mean values are bigger than 0 . 
In fact, due to the influence of factors such as noise and forming filter, the ratio between envelope variance and mean value is not strictly equal to zero. Our simulations for the ratio between variance and mean value of the five modulation modes are shown in Figure 2. Therefore, we can select 0.14 as the threshold value. When signal to noise ratio is bigger than $8 \mathrm{~dB}$, the ratios of 8PSK and QPSK are $<0.14$. For this reason, they ban be differentiated.

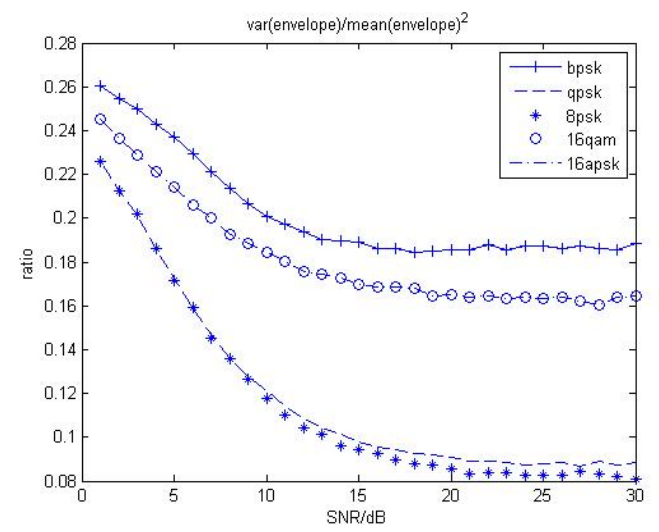

Fig.2 Ratios between envelope variances and mean values of different modulation modes

\section{Quadruplicate Spectrum}

Fourth-order spectrum refers to the fourth-order power spectrum of the signal.
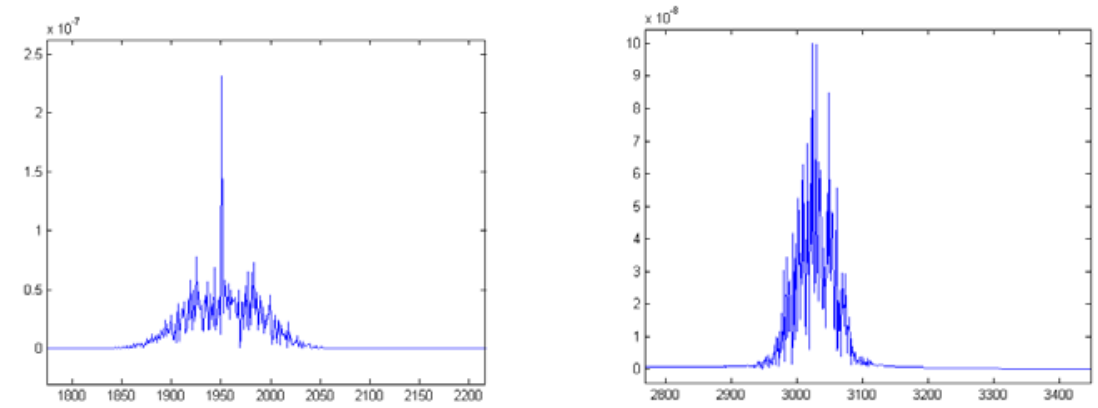

Fig.3(a) Quadruplicate spectrum of 16QAM Fig.3(b) Fourth-order spectrum of 16APSK

$\mathrm{C} 4$, the simple-frequency component estimation value in quadruplicate spectrum of definition signal is the number of single-frequency components in the quadruplicate spectrum of the signal. It can be seen from Figure 3 that the single-peak spectral line of 16APSK is basically submerged, while 16QAM still has strong single-peak spectrum line. Therefore, C4, the quadruplicate spectrum characteristics, can be used to differentiate 16APSK and 16QAM.

\section{Realization of algorithm}

From above theory and simulation analysis, it can be seen that by using signal characteristic parameters, the ratio between envelope variance and mean value, squared spectrum and quadruplicate spectrum, the five modulation modes of BPSK, 8PSK, QPSK, 16QAM and 16APSK can totally be distinguished. Specific process is as follows: 


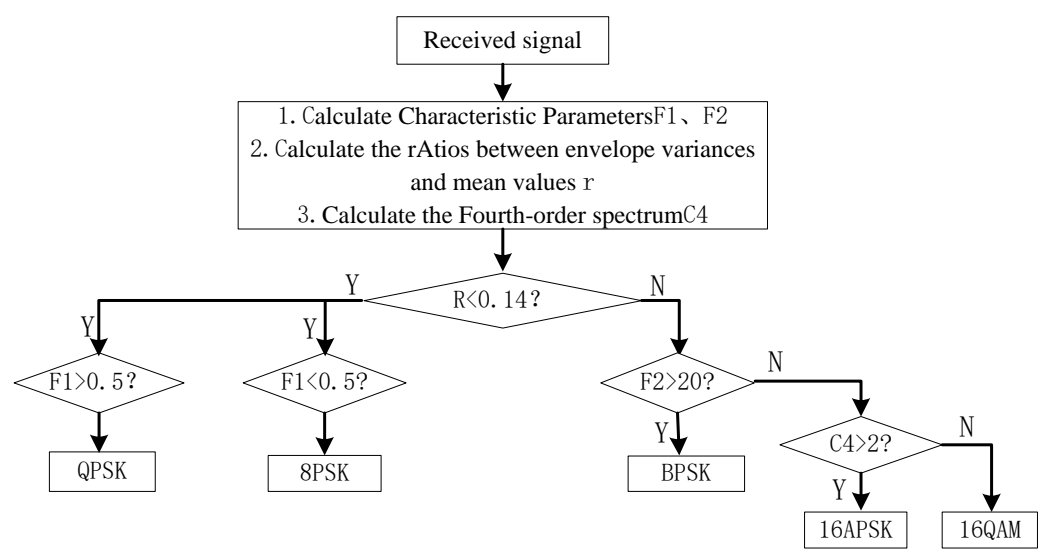

Fig.4 Procedure of modulation mode recognition algorithm 


\section{Simulation Performance}

The simulation results are shown in Figure 5:
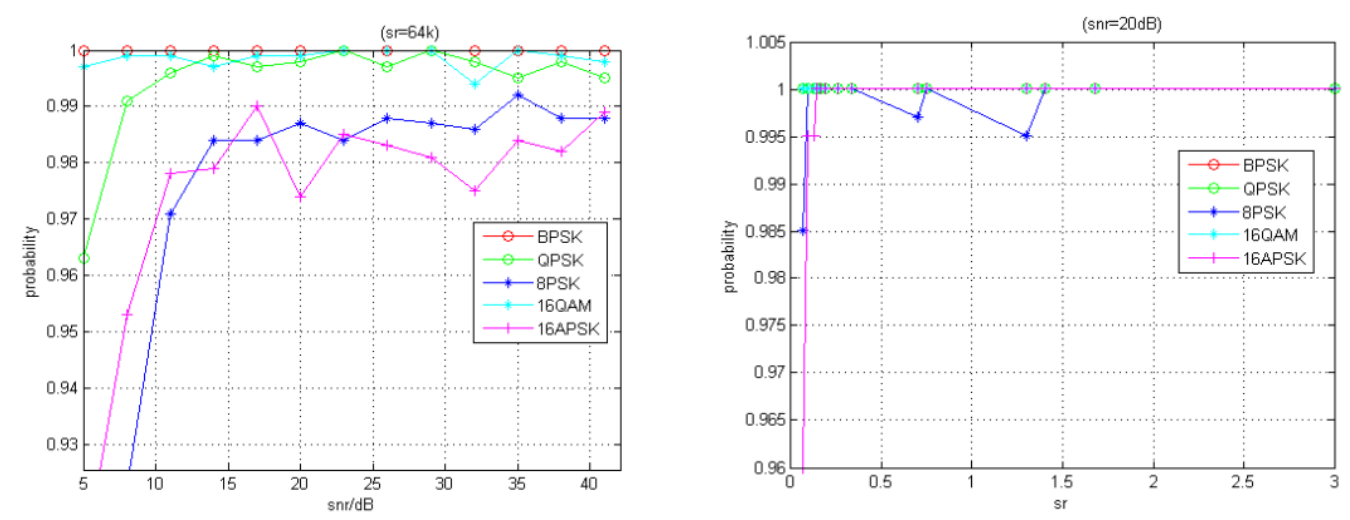

Fig.5 Recognition probability vs signal to noise ratio and symbol rate

From above figures, it can be seen that when signal to noise ratio is smaller than $10 \mathrm{~dB}$, the recognition probabilities of BPSK, QPSK, 8PSK, 16APSK and 16QAM are higher than 97\%.

\section{Conclusion}

In terms of the five commonly used modulation modes, BPSK, QPSK, 8PSK, 16APSK and 16QAM, this paper put forward a recognition algorithm combining characteristic parameters and quadruplicate spectrum. This method does not require any prior knowledge and has relatively high recognition rate, which has significant meaning for the modulation recognition of satellite communication signal. But how to remain relatively good recognition rate in relatively low signal to noise ratio still requires further research.

\section{References}

[1] E.E.Axxouz, A.K.Nandi. Algorithms for Automatic Modulation Recognition of Communication Signals. IEEE Transactions on Communications. 1998. 46(4): 431-436

[2] Xianda Zhang. Advanced Signal Processing Second Edition[M]. Beijing: Tsinghua University Press, 2002. 1-492

[3] Xiukun Huang. Estimation Technique Introduction for High-order Spectrum[J]. Journal of Northern Jiaotong University, 1991，15(2):29-44

[4] Xiaoji Wei. Research in Modulation Scheme Recognition of Digital Communication. Harbin Engineering University, 2011.30-39

[5] Huang Ying, Lei Jing. Research in Modulation Recognition of Satellite Communication [J]. Systems Engineering and Electronics, 2009,31(6):1303-1306 